\title{
The Impact of Hypo- and Hyperglycemia on Cognition and Brain Development in Young Children with Type 1 Diabetes
}

\author{
Michal Nevo-Shenker ${ }^{a}$ Shlomit Shalitin ${ }^{a, b}$ \\ a Jesse Z. and Lea Shafer Institute of Endocrinology and Diabetes, Schneider Children's Medical Center of \\ Israel, Petach Tikva, Israel; 'bSackler Faculty of Medicine, Tel Aviv University, Tel Aviv, Israel
}

\section{Keywords}

Type 1 diabetes $\cdot$ Hypoglycemia $\cdot$ Hyperglycemia $\cdot$ Brain function - Diabetes technology

\begin{abstract}
Human and experimental animal data suggest both hyperglycemia and hypoglycemia can lead to altered brain structure and neurocognitive function in type 1 diabetes (T1D). Young children with T1D are prone to extreme fluctuations in glucose levels. The overlap of these potential dysglycemic insults to the brain during the time of most active brain and cognitive development may cause cellular and structural injuries that appear to persist into adult life. Brain structure and cognition in persons with T1D are influenced by age of onset, exposure to glycemic extremes such as severe hypoglycemic episodes, history of diabetic ketoacidosis, persistent hyperglycemia, and glucose variability. Studies using brain imaging techniques have shown brain changes that appear to be influenced by metabolic abnormalities characteristic of diabetes, changes apparent at diagnosis and persistent throughout adulthood. Some evidence suggests that brain injury might also directly contribute to psychological and mental health outcomes. Neurocognitive
\end{abstract} over, impaired executive function and mental health can affect patients' adherence to treatment. This review summarizes the current data on the impact of glycemic extremes on brain structure and cognitive function in youth with T1D and the use of new diabetes technologies that may reduce these complications.

C) 2021 S. Karger AG, Basel

\section{Introduction}

Type 1 diabetes (T1D) is among the most prevalent chronic illnesses diagnosed in childhood. Diabetes management in young children is quite challenging, as they are more prone to experience extreme fluctuations in glucose levels at a time when their developing brain is undergoing wide-ranging maturational changes [1]. The unique properties of major neurodevelopmental changes during early childhood have led to the hypotheses that the developing brain may be especially vulnerable to glycemic extremes, with lasting effects on its development and associated cognition. The implementation of new diabetes technologies may reduce these complications.

Correspondence to:

Shlomit Shalitin, shalitin@ netvision.net.il 


\section{Influence of T1D on Cerebral Structure and Cognitive Function}

Brain synaptic development and metabolic demands vary throughout childhood and continue into young adulthood. Synaptic density increases rapidly during early postnatal cortical development, followed by a discrete period of synaptic pruning that typically occurs during adolescence [2]. Total brain volume increases between the ages of 1 and 6 years and by the age of 6 years reaches approximately $90 \%$ of the adult brain volume $[1,3]$. Total gray matter (GM) volume, cortical volume, and average cortical thickness all increase up to ages 9-11 years and then begin to decrease during subsequent childhood and adolescence years as synaptic pruning occurs. In contrast, myelination of white matter (WM), as reflected in WM volume, increases gradually throughout childhood, adolescence, and even early adulthood [3]. Simultaneously, the young brain has a rapidly changing metabolic demand. Between 2 and 3 months until 4 years of age, brain glucose use increases dramatically, reaching double the rate of the adult brain by the end of the first decade of life, followed by a gradual reduction toward adult levels in the next decade [4].

Research on brain structure and neurocognitive consequences of early-onset T1D and related glycemic variability is emerging. Studies comparing brain structure and neuropsychological functioning in young children with T1D to age-matched healthy controls have shown altered brain structure in children with $\operatorname{T1D}[5,6]$. A recent follow-up study by the Diabetes Research in Childhood Network (DirecNet) evaluated whether previously described brain and cognitive differences in children with T1D persist, worsen, or improve as children mature into puberty and examined associations with hyperglycemia. Results showed that total brain, GM, and WM volumes and full-scale verbal IQs were lower in the T1D group at $6,8,10$, and 12 years of age. The brain volumes and cognitive scores had a negative correlation with glycemic control (HbA1C and glucose sensor means) [7].

Ferguson et al. [8] evaluated brain structure by MRI and cognitive ability by a neuropsychological test battery, in a cohort of young adults with long-duration T1D diagnosed during childhood or adolescence. They found that lateral ventricular volumes were $37 \%$ greater and ventricular atrophy more prevalent in the early-onset T1D group as compared to the later-onset T1D group. Furthermore, in patients with early-onset diabetes, the intellectual ability and information processing ability were comparatively poorer than was observed in later-onset diabetes.
Therefore, it may be implied that neurodevelopment may be adversely affected by early-onset diabetes.

Toprak et al. [9] also evaluated brain structure and neurocognitive functions in children with T1D as compared to age-matched healthy controls. Fractional anisotropy (FA) and apparent diffusion coefficient (ADC) values were calculated for both groups. Associations between FA and ADC values and neurocognitive function tests were investigated. Subjects with diabetes demonstrated significant changes in FA and ADC values in widespread brain regions. Such changes could be early features of injury to myelinated fibers or axonal degeneration. Several studies assessing cognition in youth with early-onset diabetes have shown worse outcomes across a variety of cognitive domains - executive functions, learning, memory, and processing speed $[10,11]$.

In a cohort of young children with $\mathrm{T} 1 \mathrm{D}$, with a median diabetes duration of approximately 4 years, Cato et al. [12] observed trends toward cognitive differences relative to controls in areas of intellectual ability and executive functions after accounting for parental IQ and level of parent-reported depression. However, cognitive domain scores did not differ between the groups after an 18-month testing session and did not change significantly over the follow-up period [13]. A recent study conducted in South Australia evaluated educational outcomes in children with T1D while also comparing the effect of times from T1D diagnosis ( $<2$ years vs. 3-10 years). This study did not show differences in educational outcomes between children with T1D and controls, regardless of time since diagnosis [14].

Aye et al. [15] evaluated the correlates of cognitive abilities and WM microstructural changes in young children with T1D using diffusion tensor imaging (DTI), as compared with age- and sex-matched healthy control subjects. DTI provides a quantitative assessment of WM integrity through the measurement of diffusion of water molecules in brain tissue. They found that compared with healthy controls, children with T1D had significantly lower axial diffusivity values in the temporal and parietal lobe regions, without significant differences between groups in FA and radial diffusivity. They found a significant, positive correlation between HbAlc levels and WM structural differences (as measured by radial diffusivity). A higher HbA1c value was significantly correlated with lower overall intellectual functioning as measured by the full-scale intelligence quotient. These findings suggest that brain damage may have begun at the cellular level during the initial stages of T1D, and neurocognitive impairments may be inevitable. 
Children and adolescents with T1D are more likely to perform worse on tasks that require sustained attention, rapid processing speed, memory, and visuospatial functioning compared to their nondiabetic peers [11]. A meta-analysis showed that executive functions, including processes like working memory, attention, and response inhibition, are particularly affected [16]. Nevertheless, a recent study from DirecNet [17] reported that despite equivalent cognitive and behavioral functioning between children with T1D and age-matched nondiabetic controls, young children with T1D exhibited increased activation in executive control regions (e.g., dorsal anterior cingulate cortex, inferior frontal gyri, cerebellum, and supramarginal gyri) during performance of an attentiondemanding task. The magnitudes of these increases were significantly correlated with deficits in deactivation of the posterior node of the default mode network, suggesting a putative compensatory role of brain function in T1D, whereby higher activation in task-relevant regions acted both to offset T1D-related impairments in default mode network function and to facilitate behavioral performance levels equivalent to those of their nondiabetic peers. Another study by the DirecNet group assessed brain activation in children with T1D via functional MRI, while the children performed visuospatial working memory tasks. The study showed that children with T1D performed the tasks less accurately, yet again functional MRI indicated increased activation of relevant brain regions (frontoparietal cortex, cerebellum, and thalamus), meaning higher working memory load, presumably indicative of compensatory mechanisms needed by the T1D group. In this study, the end results showed that this compensation was not sufficiently effective to raise the T1D group's results to those of the control group. It is noteworthy that the findings were more prominent in children with younger age at T1D onset [18].

\section{The Impact of Hypoglycemia on Cognitive Function and Brain}

The young age at diagnosis of T1D contributes to significant concerns regarding hypoglycemia. For different reasons, including lack of expressive language skills and cognitive immaturity, young children may be unable to reliably detect and/or report early symptoms of hypoglycemia.

Several studies of patients with childhood-onset T1D documented associations between severe hypoglycemia (with seizures or loss of consciousness) and brain chang-

Cognition and Brain Development in

Young Children with Diabetes es. Perantie et al. [19] found significant differences between young patients with T1D and healthy control groups with regard to GM or WM. However, within the diabetic group, a history of severe hypoglycemia was associated with smaller GM volume in the left superior temporal region. The same group, in a prospective study, reported that severe hypoglycemia experienced between brain imaging scans was related to reduced WM volume growth in the parietal occipital cortex over a 2-year time period [20]. Musen et al. [21] found, in patients with T1D with early-onset diabetes, lower levels of GM density associated with worse glycemic control and higher frequency of recurrent severe, hypoglycemic events. However, another retrospective study found no significant effects of severe hypoglycemia on WM integrity across the brain in T1D youth [22]. Clearly, there is a lack of consistent findings of the impact of previous severe hypoglycemia on regional or whole brain volumes.

Many studies evaluated the magnitude and pattern of cognitive dysfunction in children with T1D and possible effects associated with severe hypoglycemia and poor cognitive outcomes [23-26]. A meta-analysis of 10 studies described the impact of severe hypoglycemic episodes on cognitive function [27]. The severe hypoglycemia group included patients who had at least one severe hypoglycemic episode, and the nonhypoglycemia group included those with no hypoglycemic episodes. The performance of T1D participants with severe hypoglycemia $(n=347)$ was compared to those without hypoglycemic episodes $(n=364)$. Children with severe hypoglycemic episodes were somewhat more impaired in overall cognition than children without hypoglycemia and showed slightly lower performance in memory. In other cognitive domains, the effects were not significant. The results indicated that children with severe hypoglycemia showed significantly poorer performance in overall cognition. These findings are consistent with previous reviews and developmental cognitive studies in children with recurrent hypoglycemic episodes [24, 25].

An additional sub-meta-analysis explored differences in cognitive domains between children with early-onset (up to 7 years of age) compared with late-onset severe hypoglycemia [27]. Children with early-onset severe hypoglycemia performed more poorly than those with lateonset severe hypoglycemia in overall cognition. These findings indicate that severe hypoglycemia experienced early in development might be more harmful to cognitive performance than severe hypoglycemia later in life. In the latter meta-analysis [27], a subgroup analysis evaluated the cross-sectional and longitudinal studies. Cross-sec- 
tional studies showed that the effects of severe hypoglycemia on cognitive dysfunction were significant; however, longitudinal studies suggested that severe hypoglycemia episodes were not associated with cognitive dysfunction. Hence, severe hypoglycemia might be a plausible cause of cognitive decline in children with T1D. Thus, more longitudinal studies are needed to fully address the long-term effects of severe hypoglycemia on cognitive dysfunction.

\section{The Impact of Hyperglycemia and Glucose Variability on the Brain and Cognitive Function}

Chronic hyperglycemia exposure may also affect the brain, targeting both GM and WM volume in youth with T1D. Using structural MRI, Marzelli and colleagues [28] found that young children with T1D with a history of significant hyperglycemia exhibited decreased GM volume in key brain regions associated with cognitive capacities, compared to healthy control participants. The association between glycemic variability, particularly hyperglycemia, and cognitive function was more pronounced in young children with earlier onset and longer duration of diabetes, further highlighting the brain's vulnerability in this age group. In this same sample from the DirecNet study, Cato and colleagues [29] reported trending associations among executive functioning, learning/memory, and hyperglycemia, suggesting that the structural brain changes in youth with diabetes have a subtle yet measurable impact on cognition as early as 2 years after T1D onset. These data suggest that glycemic dysregulation or variability has significant implications for young children and brain structure and function.

A large cohort of young children (age 4 to $<10$ years) with T1D from the DirecNet study was longitudinally compared with an age-matched healthy control group [5, 30]. The results demonstrated that early-onset diabetes significantly affected the development of total and regional GM and WM volumes, with differences between groups enhanced over time. Remarkably, in the diabetic group, the slower growth was most strongly associated with hyperglycemia and glycemic variability, as measured by several metrics, including glycated hemoglobin and extensive quarterly data from continuous glucose monitoring (CGM).

Another report from the DirecNet study analyzed longitudinal trajectories of WM using DTI [31]. It was found that axial diffusivity was lower in children with diabetes at baseline and 18-month follow-up, indicating that dif- ferences in WM microstructure persisted over time. Within the diabetes group, lower exposure to hyperglycemia, averaged over time since diagnosis, was associated with higher FA, which in turn was positively correlated with performance and full-scale IQ. These studies provide strong evidence that the developing brain is a vulnerable target for hyperglycemia with altered WM development, which may contribute to the mild cognitive deficits in this population.

Several mechanisms for the observed slower total and regional brain growth in children with T1D may be operative. Chronic hyperglycemia can lead to formation of advanced glycation end products and their receptors, nuclear factor- $\kappa \mathrm{B}$, greater increased oxidative stress, and even neurodegeneration [32-34]. These glycemic correlations support the notion that increased glucose variability may damage developing neurons and myelin in children with T1D and are congruent with observations from streptozotocin-induced diabetes animal models that show in vivo degenerative changes of neurons and glia, disarrangement of myelin sheaths, and reduced myelin content with hyperglycemia [34]. Changes in brain sphingolipid composition (ceramides and sphingomyelin) induced by hyperglycemia may also provoke membrane rearrangements in some cell populations, which can disturb cellular signaling and cause brain tissue damage [35]. The ultimate mechanism of the observed changes is likely multifactorial.

Central nervous system insult to children with earlyonset diabetes may have a delayed, progressive, and $\mathrm{cu}-$ mulative impact on neuropsychological outcomes and cognition over time. These outcomes might be subtle in terms of cognition but appear to persist into adult life [36]. Concurrent baseline neuroimaging analysis performed by DirecNet supports the hypothesis that WM disruption has occurred at this early stage in disease progression, with a significant association between WM structure and cognitive ability in children with T1D but not in control subjects $[29,30]$.

Kirchhoff and colleagues [37] examined visual-spatial ability, as well as memory and processing speed, among children aged $4-16$ years with T1D $(n=119)$ as compared to their healthy siblings $(n=59)$ over a 5 -year period. Children with T1D showed worse performance on visualspatial ability and memory tasks. An inverse correlation was seen between hyperglycemia and processing speed, where individual improvement of glycemic control (as seen between time points) correlated with improved visual-spatial ability and processing speed [37].

Glycemic extremes are associated with poorer overall cognition, as well as slightly lower performance in mem- 
Table 1. The main findings on the effect of hypo- and hyperglycemia on the brain

\begin{tabular}{|c|c|}
\hline Effect of hypoglycemia on the brain & Effect of hyperglycemia on the brain \\
\hline GM & $G M$ \\
\hline Lower levels of GM density (in early-onset DM) [21] & Less growth of cortical GM [5] \\
\hline Smaller volume in left superior temporal region [19] & Decreased volume $[7,28]$ \\
\hline$W M$ & $W M$ \\
\hline Less growth of WM volume in the cortex and cerebellum [5] & Lower volume [7] \\
\hline Reduced volume growth in the parietal occipital cortex [20] & Decreased WM development $[5,30]$ \\
\hline No significant effect on WM integrity [22] & Altered WM microstructure [31] \\
\hline \multirow{10}{*}{$\begin{array}{l}\text { Cognitive function } \\
\text { Poor performance in overall cognition, lower memory } \\
\text { performance }[24,25,27] \\
\text { Poorer overall performance in early-onset diabetes } \\
\text { (age }<7 \text { years) [27] }\end{array}$} & Cognitive function \\
\hline & Lower cognitive scores $[7,28,31]$ \\
\hline & Lower overall intellectual function [15] \\
\hline & Impaired executive function $[29,42]$ \\
\hline & Impaired learning/memory $[29,37,38]$ \\
\hline & Visual-spatial ability [37] \\
\hline & Others \\
\hline & Slower hippocampus growth [6] \\
\hline & Formation of advanced glycation end products and increased \\
\hline & Cell membrane rearrangements and cellular signaling disturbance \\
\hline
\end{tabular}

The numbers denote the relevant references. GM, gray matter; WM, white matter.

ory [38]. A history of chronic hyperglycemia appears to be more injurious than previously suspected. Neurocognitive deficits manifest across multiple cognitive domains, including executive function and speed of information processing. One study showed that glucose variability might have a greater adverse impact on the developing brain than either prolonged high or low glucose levels [39]. There were 2 possible mechanisms through which blood glucose variability leads to cognitive dysfunction, according to previous studies. One showed that glycemic variability was associated with increased production of reactive oxygen species that could damage the central nervous system [40]. Another mechanism showed that oscillating glucose could have a more toxic impact on oxidative stress generation than constant high glucose, which might lead to mitochondrial dysfunction and neuronal cell damage [41].

Subtle brain injury might directly contribute to psychological and mental health outcomes. Impaired executive function and mental health, in turn, could affect patients' adherence to and their ability to make adaptive lifestyle choices [42]. Table 1 presents the main findings on the effect of hypo- and hyperglycemia on the brain.

Cognition and Brain Development in

Young Children with Diabetes

\section{The Impact of DKA on the Brain and Cognitive Function}

An episode of diabetic ketoacidosis (DKA) has acute structural effects on the brain, such as clinical and subclinical cerebral edema [43], both at time of diagnosis and with regard to MRI-associated volume and DTI changes 3 months after diagnosis [44]. DKA has been reported to acutely affect the diffusivity of the thalamus in a pediatric population [45]. DKA can lead to brain swelling and reduced cerebral blood flow, with potential long-term impact on brain development [46].

A history of DKA has been associated with long-term adverse cognitive effects $[47,48]$. Subtle learning problems, emotional problems, and poor concentration have been reported in children after an episode of DKA, and evidence suggests long-lasting decreases in memory function in school-aged children (7-16 years) for years after a DKA episode [47].

A recent study [49] evaluated whether severity of clinical presentation (presence of DKA and HbA1c at diagnosis) contributed to differences in cognition and brain structure. The study evaluated newly diagnosed youth with T1D, using MRI scans, comparing them to healthy 
siblings without diabetes. Results showed that after controlling for age, sex, and multiple comparisons, the T1D group had lower volume in the left temporal-parietal-occipital cortex compared with controls. Within the T1D group, DKA at presentation was associated with lower radial, axial, and mean diffusivity throughout major WM tracts. Higher HbAlc was associated with lower hippocampal, thalamic, and cerebellar WM volumes, lower right posterior parietal cortical thickness, and greater right occipital cortical thickness. These data suggest that severity of clinical presentation is an important factor in predicting brain structural differences in youth with T1D.

Another recent study among young children with T1D who participated in the DirecNet study examined whether a history of DKA was associated with changes in longitudinal cognitive and brain development as evaluated by cognitive testing and brain MRI scans at baseline and 18 months. They found that the moderate/severe DKA group gained more total and regional WM and GM volume over the observed 18 months as compared with the none/mild group. When matched by age at time of enrollment and average $\mathrm{HbA}_{1 \mathrm{c}}$ during the 18-month interval, participants who had a history of moderate/severe DKA compared with none/mild DKA were observed to have significantly lower cognitive scores [50].

Recently, Ghetti et al. [51] evaluated whether a DKA episode, either at diabetes diagnosis or later in the course of the disease, affected cognitive function. Their study is unique in its prospective nature and size, including 758 children with DKA and 376 controls (T1D without DKA) aged 6-18 years. They showed that moderate/severe DKA correlated with lower IQ and success rates on recall tasks and that repeated DKA episodes and poorer glycemic control correlated with lower IQ and recall [51].

\section{The Implementation of New Diabetes Technologies to Reduce Hypo- and Hyperglycemia and Their Impact on Cognition and Brain Development}

The glycemic target of a glycated hemoglobin level of $<7 \%$ (as recommended by the American Diabetes Association and International Society of Pediatric Diabetes) is attained by $<20 \%$ of children with T1D [52]. Advanced technologies have become an integral part of T1D management with potential to improve glycemic outcomes and its consequences and quality of life. They include continuous subcutaneous insulin delivery, CGM, sensoraugmented pumps (SAP), predictive low glucose insulin suspension (PLGS), and closed-loop systems that auto- mate insulin delivery in a glucose-responsive manner (artificial pancreas).

CGM use among very young children is feasible and enables reduction of both time spent in hypoglycemia and glucose variability. A recent study by the Strategies to Enhance New CGM Use in Early Childhood study group (SENCE) evaluated the effects of CGM with or without family behavioral intervention on glycemic outcomes in young children (2-8 years). The study showed improvement in critical glycemic measures in the CGM group (time spent in hypoglycemia, number of severe hypoglycemic events, and glucose variability), emphasizing the yield of CGM use in this young age group [53]. A Slovenian population-based cohort assessing the correlation between CGM use and glucose variability in young children (age $<8$ years, $n=40$ ) over a 5 -year time period showed that both standard deviation of mean glucose and coefficient of variance were lower during periods of CGM use [54].

It has been shown that continuous subcutaneous insulin delivery treatment reduces the frequency of severe hypoglycemia compared with multiple daily injection treatment [55]. The addition of a CGM, known as SAP treatment, has the potential to further limit the duration and severity of hypoglycemia as some systems can detect and in some cases act on impending and prevailing low blood glucose levels, without significantly affecting metabolic control or causing DKA $[56,57]$.

The use of the SmartGuard algorithm built into the MiniMed 640G demonstrated that the system can prevent severe hypoglycemia in children and adolescents, by decreasing both the duration of hypoglycemic events and the intensity of hypoglycemia, with significantly fewer hypoglycemic events [58]. The protective effect of PLGS in the Medtronic 640G was also shown in a randomized controlled study in children and adolescents $(n=100)$ who endured a significantly reduced number and duration of hypoglycemic events [59]. Similar results were also shown in a recent study in very young children (3-6 years of age, $n=44)$, in whom the PLGS enabled reaching a time in range (TIR) of $82 \%$ [60].

Current studies of home use of day-and-night closedloop insulin delivery systems in very young children with T1D proved the system was feasible and safe, with increased time in the target glucose levels range and without related severe hypoglycemia or DKA events [61, 62]. A recent short pilot study evaluated a modified investigational version of the Tandem $t: s \lim \mathrm{X}^{2}$ Control-IQ system in very young children ( $2-5$ years old) in both a supervised and home setting with unrestricted meals and snacks. The system significantly improved TIR compared to baseline, 
with $<6 \%$ hypoglycemia and $<40 \%$ hyperglycemia in $83 \%$ of participants compared to $33 \%$ prior to system use [63].

Another study evaluating the efficacy and safety of closed-loop systems was conducted among children ( $>6$ years of age) as well as adolescents and adults. The study aimed to imitate free-living conditions with unrestricted meals and daily physical activity. The study showed that even under free-living conditions, the TIR was significantly improved among children with fewer episodes of hypoglycemia among this age group [64].

The Omnipod 5, a tubeless automated insulin delivery system, has also been assessed for safety and efficacy among children. The use of the system was compared to standard therapy among children aged $6-13$ over a 2 -week period. The participants used the system with both prespecified glucose targets $(130,140$, and $150 \mathrm{mg} / \mathrm{dL}, 9$ days total) and free choice of glucose targets (110-150 mg/dL). Study results showed a significantly higher TIR among children during both the free-choice period and the 110, 130 , and $140 \mathrm{mg} / \mathrm{dL}$ target as compared with standard therapy. The TIR did not come at the expense of episodes of hypoglycemia or other adverse events [65].

A multicenter, multinational, randomized cross-over study among very young children (ages 1-7 years) is currently underway. The study aims to evaluate whether the Cambridge closed-loop algorithm implemented in the CamAPS FX app will increase TIR over 16 weeks as compared with SAP therapy. Besides, the very young age group the study is also unique in the assessment of quality of life parameters as well as the glycemic measures such as $\mathrm{HbA1C}$, TIR, and glucose variation [66].

In order to try to improve their children's and family's quality of life, more parents are implementing do-ityourself closed-loop systems. A recent study [67] retrospectively examined the efficacy and safety of such a system, AndroidAPS, in preschool and school-aged children (2 groups, 3-7 years and 8-14 years). After switching from SAP to AndroidAPS, both groups showed increased TIR and lower HbA1C levels, with no episodes of severe hypoglycemia or DKA.

\section{Conclusions}

The treatment of T1D in young children is challenging for both parents and caregivers. Hypoglycemia represents a burden for the wellbeing of young children with T1D and may have a serious impact on their brain development and cognitive function. However, in recent years, several studies have reported that chronic hyperglycemia exposure and increased glucose variability may also have significant implications for brain structure and function in young children with T1D. Therefore, improved and novel technologies in insulin delivery and glucose monitoring implemented in recent years aim to enhance the flexibility of care and optimize glycemic control, while trying to enable a "normal" life with decreased damage to the developing brain and neurocognitive function.

\section{Acknowledgment}

The authors wish to thank Debby Mir for the editorial assistance.

\section{Conflict of Interest Statement}

The authors have no conflicts of interest relevant to this article to disclose.

\section{Funding Sources}

This research did not receive any specific grant from funding agencies in the public, commercial, or not-for-profit sectors.

\section{Author Contributions}

S.S. and M.N. were responsible for drafting the article and revising it critically for important intellectual content. Both authors read and approved the final manuscript.

\section{References}

Cognition and Brain Development in

Young Children with Diabetes
1 Giedd JN, Rapoport JL. Structural MRI of pediatric brain development: what have we learned and where are we going? Neuron. 2010 Sep;67(5):728-34.

2 Bullmore E, Sporns O. The economy of brain network organization. Nat Rev Neurosci. 2012 Apr;13(5):336-49.

3 Tamnes CK, Ostby Y, Fjell AM, Westlye LT, Due-Tønnessen P, Walhovd KB. Brain matu- ration in adolescence and young adulthood: regional age-related changes in cortical thickness and white matter volume and microstructure. Cereb Cortex. 2010 Mar;20:534-48.

4 Kuzawa CW, Chugani HT, Grossman LI, Lipovich L, Muzik O, Hof PR, et al. Metabolic costs and evolutionary implications of human brain development. Proc Natl Acad Sci U S A. 2014 Aug;111:13010-5. 
5 Mazaika PK, Weinzimer SA, Mauras N, Buckingham B, White $\mathrm{NH}$, Tsalikian E, et al. Variations in brain volume and growth in young children with type 1 diabetes. Diabetes. 2016 Feb;65(2):476-85.

6 Foland-Ross LC, Reiss AL, Mazaika PK, Mauras N, Weinzimer SA, Aye T, et al. Longitudinal assessment of hippocampus structure in children with type 1 diabetes. Pediatr Diabetes. 2018 Apr;19(6):1116-23.

7 Mauras N, Buckingham B, White NH, Tsalikian E, Weinzimer SA, Jo B, et al. Impact of type 1 diabetes in the developing brain in children: a longitudinal study. Diabetes Care. 2021 Apr;44(4):983-92.

8 Ferguson SC, Blane A, Wardlaw J, Frier BM, Perros P, McCrimmon RJ, et al. Influence of an early-onset age of type 1 diabetes on cerebral structure and cognitive function. Diabetes Care. 2005 Jun;28(6):1431-7.

9 Toprak H, Yetis H, Alkan A, Filiz M, Kurtcan S, Aralasmak A, et al. Relationships of DTI findings with neurocognitive dysfunction in children with Type 1 diabetes mellitus. $\mathrm{Br} \mathrm{J}$ Radiol. 2016 Jan;89(1059):20150680.

10 Ly TT, Anderson M, McNamara KA, Davis EA, Jones TW. Neurocognitive outcomes in young adults with early-onset type 1 diabetes: a prospective follow-up study. Diabetes Care. 2011 Oct;34(10):2192-7.

11 Gaudieri PA, Chen R, Greer TF, Holmes CS Cognitive function in children with type $1 \mathrm{di}-$ abetes: a meta-analysis. Diabetes Care. 2008 Sep;31(9):1892-7.

12 Cato MA, Mauras N, Ambrosino J, Bondurant A, Conrad AL, Kollman C, et al. DirecNet. Cognitive functioning in young children with type 1 diabetes. J Int Neuropsychol Soc. 2014 Feb;20(2):238-47.

13 Cato MA, Mauras N, Mazaika P, Kollman C, Cheng P, Aye T, et al. DirecNet. Longitudinal evaluation of cognitive functioning in young children with type 1 diabetes over 18 months. J Int Neuropsychol Soc. 2016 Mar;22(3):293302.

14 Begum M, Chittleborough C, Pilkington R, Mittinty M, Lynch J, Penno M, et al. Educational outcomes among children with type 1 diabetes: whole-of-population linked-data study. Pediatr Diabetes. 2020 Nov;21:135361.

15 Aye T, Reiss AL, Kesler S, Hoang S, Drobny J, Park Y, et al. The feasibility of detecting neuropsychologic and neuroanatomic effects of type 1 diabetes in young children. Diabetes Care. 2011 Jul;34(7):1458-62.

16 Broadley MM, White MJ, Andrew B. A systematic review and meta-analysis of executive function performance in type 1 diabetes mellitus. Psychosom Med. 2017 Jul/Aug;79(6): 684-96.

17 Foland-Ross LC, Buckingam B, Mauras N, Arbelaez AM, Tamborlane WV, Tsalikian E, et al. DirecNet. Executive task-based brain function in children with type 1 diabetes: an observational study. PLoS Med. 2019 Dec; 16(12):e1002979.
18 Foland-Ross LC, Tong G, Mauras N, Cato A, Aye T, Tansey M, et al. DirecNet. Brain function differences in children with type 1 diabetes: an fMRI study of working memory. Diabetes. 2020 Aug;69(8):1770-8.

19 Perantie DC, Wu J, Koller JM, Lim A, Warren SL, Black KJ, et al. Regional brain volume differences associated with hyperglycemia and severe hypoglycemia in youth with type 1 diabetes. Diabetes Care. 2007 Sep;30(9):2331-7.

20 Perantie DC, Koller JM, Weaver PM, Lugar HM, Black KJ, White NH, et al. Prospectively determined impact of type 1 diabetes on brain volume during development. Diabetes. 2011 Nov;60(11):3006-14.

21 Musen G, Lyoo IK, Sparks CR, Weinger K, Hwang J, Ryan CM, et al. Effects of type 1 diabetes on gray matter density as measured by voxel-based morphometry. Diabetes. 2006 Feb;55:326-33.

22 Antenor-Dorsey JA, Meyer E, Rutlin J, Perantie DC, White NH, Arbelaez AM, et al. White matter microstructural integrity in youth with type 1 diabetes. Diabetes. 2013 Feb;62: 581-9.

23 Blasetti A, Chiuri RM, Tocco AM, Di Giulio C, Mattei PA, Ballone E, et al. The effect of recurrent severe hypoglycemia on cognitive performance in children with type 1 diabetes: a meta-analysis. J Child Neurol. 2011 Nov; 26(11):1383-91.

24 Hershey T, Lillie R, Sadler M, White NH. Severe hypoglycemia and long-term spatial memory in children with type 1 diabetes mellitus: a retrospective study. J Int Neuropsychol Soc. 2003 Jul;9(5):740-50.

25 Hershey T, Perantie DC, Warren SL, Zimmerman EC, Sadler M, White NH. Frequency and timing of severe hypoglycemia affects spatial memory in children with type 1 diabetes. Diabetes Care. 2005 Oct;28:2372-7.

26 Perantie DC, Lim A, Wu J, Weaver P, Warren SL, Sadler M, et al. Effects of prior hypoglycemia and hyperglycemia on cognition in children with type 1 diabetes mellitus. Pediatr Diabetes. 2008 Apr;9(2):87-95.

27 He J, Ryder AG, Li S, Liu W, Zhu X. Glycemic extremes are related to cognitive dysfunction in children with type 1 diabetes: a meta-analysis. J Diabetes Investig. 2018 Nov;9(6):134253.

28 Marzelli MJ, Mazaika PK, Barnea-Goraly N, Hershey T, Tsalikian E, Tamborlane W, et al. DirecNet. Neuroanatomical correlates of dysglycemia in young children with type 1 diabetes. Diabetes. 2014 Jan;63:343-53.

29 Cato MA, Mauras N, Ambrosino J, Bondurant A, Conrad AL, Kollman C, et al. Cognitive functioning in young children with type 1 diabetes. J Int Neuropsychol Soc. 2014 Feb; 20:238-47.

30 Mauras N, Mazaika P, Buckingham B, Weinzimer S, White NH, Tsalikian E, et al. Longitudinal assessment of neuroanatomical and cognitive differences in young children with type 1 diabetes: association with hyperglycemia. Diabetes. 2015 May;64(5):1770-9.
31 Fox LA, Hershey T, Mauras N, Arbeláez AM, Tamborlane WV, Buckingham B, et al. DirecNet. Persistence of abnormalities in white matter in children with type 1 diabetes. Diabetologia. 2018 Apr;61(7):1538-154.

32 Aragno M, Mastrocola R, Medana C, Restivo F, Catalano MG, Pons N, et al. Up-regulation of advanced glycated products receptors in the brain of diabetic rats is prevented by antioxidant treatment. Endocrinology. 2005 Dec; 146:5561-7.

33 King GL, Loeken MR. Hyperglycemia-induced oxidative stress in diabetic complications. Histochem Cell Biol. 2004 Oct;122: 333-8.

34 Wang X, Yu S, Hu JP, Wang CY, Wang Y, Liu $\mathrm{HX}$, et al. Streptozotocin-induced diabetes increases amyloid plaque deposition in $\mathrm{AD}$ transgenic mice through modulating AGEs/ RAGE/NF-kB pathway. Int J Neurosci. 2014 Aug;124:601-8.

35 Fiedorowicz A, Prokopiuk S, Zendzian-Piotrowska M, Chabowski A, Car H. Sphingolipid profiles are altered in prefrontal cortex of rats under acute hyperglycemia. Neuroscience. 2014 Jan;256:282-91.

36 Jacobson AM, Ryan CM, Cleary PA, Waberski BH, Weinger K, Musen G, et al. Biomedical risk factors for decreased cognitive functioning in type 1 diabetes: an 18 year followup of the diabetes control and complications trial (DCCT) cohort. Diabetologia. $2011 \mathrm{Feb}$; 54(2):245-55.

37 Kirchhoff BA, Jundt DK, Doty T, Hershey T. A longitudinal investigation of cognitive function in children and adolescents with type 1 diabetes mellitus. Pediatr Diabetes. 2017 Sep;18(6):443-9.

38 He J, Ryder AG, Li S, Liu W, Zhu X. Glycemic extremes are related to cognitive dysfunction in children with type 1 diabetes: a meta-analysis. J Diabetes Investig. 2018 Nov;9(6):134253.

39 Northam EA, Cameron FJ. Understanding the diabetic brain: new technologies but old challenges. Diabetes. 2013 Feb;62:341-2.

40 Piconi L, Quagliaro L, Assaloni R, Da Ros R, Maier A, Zuodar G, et al. Constant and intermittent high glucose enhances endothelial cell apoptosis through mitochondrial superoxide overproduction. Diabetes Metab Res Rev. 2006 May-Jun;22:198-203.

41 Arbelaez AM, Semenkovich K, Hershey T. Glycemic extremes in youth with T1DM: effects on the developing brain's structural and functional integrity. Pediatr Diabetes. 2013 Dec;14:541-53.

42 Cameron FJ, Northam EA, Ryan CM. The effect of type 1 diabetes on the developing brain. Lancet Child Adolesc Health. 2019 Jun;3(6): 427-36.

43 Siller AF, Lugar H, Rutlin J, Koller JM, Semenkovich $\mathrm{K}$, White $\mathrm{NH}$, et al. Severity of clinical presentation in youth with type 1 diabetes is associated with differences in brain structure. Pediatr Diabetes. 2017 Dec;18:68695. 
44 Jessup AB, Grimley MB, Meyer E, Passmore GP, Belger A, Hoffman WH, et al. Effects of diabetic ketoacidosis on visual and verbal neurocognitive function in young patients presenting with new-onset type 1 diabetes. J Clin Res Pediatr Endocrinol. 2015 Sep;7:20310.

45 Vavilala MS, Marro KI, Richards TL, Roberts JS, Curry P, Pihoker C, et al. Change in mean transit time, apparent diffusion coefficient, and cerebral blood volume during pediatric diabetic ketoacidosis treatment. Pediatr Crit Care Med. 2011 Nov;12(6):e344-9.

46 Glaser N, Ngo C, Anderson S, Yuen N, Trifu A, O'Donnell M. Effects of hyperglycemia and effects of ketosis on cerebral perfusion, cerebral water distribution, and cerebral metabolism. Diabetes. 2012 Jul;61:1831-7.

47 Ghetti S, Lee JK, Sims CE, Demaster DM, Glaser NS. Diabetic ketoacidosis and memory dysfunction in children with type 1 diabetes. J Pediatr. 2010 Jan;156:109-14.

48 Semenkovich K, Bischoff A, Doty T, Nelson S, Siller AF, Hershey T, et al. Clinical presentation and memory function in youth with type 1 diabetes. Pediatr Diabetes. 2016 Nov; 17: 492-9.

49 Siller AF, Lugar H, Rutlin J, Koller JM, Semenkovich K, White NH, et al. Severity of clinical presentation in youth with type 1 diabetes is associated with differences in brain structure. Pediatr Diabetes. 2017 Dec;18(8): 686-95.

50 Aye T, Mazaika PK, Mauras N, Marzelli MJ, Shen H, Hershey T, et al. DirecNet. Impact of early diabetic ketoacidosis on the developing brain. Diabetes Care. 2019 Mar;42(3):443-9.

51 Ghetti S, Kuppermann N, Rewers A, Myers SR, Schunk JE, Stoner MJ, et al. Pediatric emergency care applied research network (PECARN) DKA FLUID Study Group. Cognitive function following diabetic ketoacidosis in children with new-onset or previously diagnosed type 1 diabetes. Diabetes Care. 2020 Nov;43(11):2768-75.

52 Foster NC, Beck RW, Miller KM, Clements MA, Rickels MR, DiMeglio LA, et al. State of type 1 diabetes management and outcomes from the T1D Exchange in 2016-2018. Diabetes Technol Ther. 2019 Feb;21:66-72.
53 DiMeglio L, Kanapka LG, DeSalvo DJ, Hilliard ME, Laffel LM, Tamborlane WV, et al. Strategies to enhance new CGM Use in Early Childhood (SENCE) Study Group. A randomized clinical trial assessing continuous glucose monitoring (CGM) use with standardized education with or without a family behavioral intervention compared with fingerstick blood glucose monitoring in very young children with type 1 diabetes. Diabetes Care. 2021 Feb;44(2):464-72.

54 Dovc K, Cargnelutti K, Sturm A, Selb J, Bratina N, Battelino T. Continuous glucose monitoring use and glucose variability in preschool children with type 1 diabetes. Diabetes Res Clin Pract. 2019;147:76-80.

55 Johansen A, Kanijo B, Fredheim S, Olsen B, Hertz B, Lauridsen MH, et al. Prevalence and predictors of severe hypoglycemia in Danish children and adolescents with diabetes. Pediatr Diabetes. 2015 Aug;16(5):354-60.

56 Forlenza GP, Pinhas-Hamiel O, Liljenquist DR, Shulman DI, Bailey TS, Bode BW, et al. Safety evaluation of the MiniMed 670G system in children 7-13 years of age with type 1 diabetes. Diabetes Technol Ther. 2019 Jan; 21(1):11-9.

57 Forlenza GP, Li Z, Buckingham BA, Pinsker JE, Cengiz E, Wadwa RP, et al. Predictive lowglucose suspend reduces hypoglycemia in adults, adolescents, and children with type 1 diabetes in an at-home randomized crossover study: results of the PROLOG trial. Diabetes Care. 2018 Oct;41(10):2155-61.

58 Biester T, Kordonouri O, Holder M, Remus $\mathrm{K}$, Kieninger-Baum D, Wadien $\mathrm{T}$, et al. "Let the algorithm do the work": reduction of hypoglycemia using sensor-augmented pump therapy with predictive insulin suspension (SmartGuard) in pediatric type 1 diabetes patients. Diabetes Technol Ther. 2017 Mar; 19(3):173-82.

59 Battelino T, Nimri R, Dovc K, Phillip M, Bratina N. Prevention of hypoglycemia with predictive low glucose insulin suspension in children with type 1 diabetes: a randomized controlled trial. Diabetes Care. 2017 Jun;40(6): 764-70.

60 Gaweł WB, Deja G, Kamińska H, Tabor A, Skała-Zamorowska E, Jarosz-Chobot P. How does a predictive low glucose suspend (PLGS) system tackle pediatric lifespan challenges in diabetes treatment? Real world data analysis. Pediatr Diabetes. 2020 Mar;21(2):280-7.

61 Tauschmann M, Allen JM, Nagl K, Fritsch M, Yong J, Metcalfe E, et al. Home use of dayand-night hybrid closed-loop insulin delivery in very young children: a multicenter, 3-week, randomized trial. Diabetes Care. 2019 Apr; 42(4):594-600.

62 Kanapka LG, Wadwa RP, Breton MD, Ruedy KJ, Ekhlaspour L, Forlenza GP, et al. Extended use of the control-IQ closed-loop control system in children with type 1 diabetes. Diabetes Care. 2021 Feb;44(2):473-8.

63 Ekhlaspour L, Schoelwer MJ, Forlenza GP, Deboer MD, Norlander L, Hsu L, et al. Safety and performance of the tandem $\mathrm{t}$ :slim X2 with control-IQ automated insulin delivery system in toddlers and preschoolers. Diabetes Technol Ther. 2021 May;23(5):384-91.

64 Sherr JL, Buckingham BA, Forlenza GP, Galderisi A, Ekhlaspour L, Wadwa RP, et al. Safety and performance of the Omnipod hybrid closed-loop system in adults, adolescents, and children with type 1 diabetes over 5 days under free-living conditions. Diabetes Technol Ther. 2020;22(3):174-84.

65 Forlenza GP, Buckingham BA, Brown SA, Bode BW, Levy CJ, Criego AB, et al. First outpatient evaluation of a tubeless automated insulin delivery system with customizable glucose targets in children and adults with type 1 diabetes. Diabetes Technol Ther. 2021;23:6.

66 Fuchs J, Allen JM, Boughton CK, Wilinska ME, Thankamony A, de Beaufort C, et al. Assessing the efficacy, safety and utility of closed-loop insulin delivery compared with sensor-augmented pump therapy in very young children with type 1 diabetes (KidsAP02 study): an open-label, multicentre, multinational, randomised cross-over study protocol. BMJ Open. 2021;11(2):e042790.

67 Petruzelkova L, Jiranova P, Soupal J, Kozak M, Plachy L, Neuman V, et al. Pre-school and school-aged children benefit from the switch from a sensor-augmented pump to an AndroidAPS hybrid closed loop: a retrospective analysis. Pediatr Diabetes. 2021;22(4):594604
Cognition and Brain Development in Young Children with Diabetes
Horm Res Paediatr 2021;94:115-123

DOI: $10.1159 / 000517352$ 